Colditz JB, Welling J, Smith NA, James AE, Primack BA. (2017). World Vaping Day: Contextualizing vaping culture in online social media using a mixed methods approach. Journal of Mixed Methods Research. doi: $10.1177 / 1558689817702753$

\title{
World Vaping Day: Contextualizing Vaping Culture in Online Social Media Using a Mixed Methods Approach
}

\author{
Jason B. Colditz, $\mathrm{MEd}^{\mathrm{a}}$ \\ Joel Welling, $\mathrm{PhD}^{\mathrm{b}}$ \\ Noah A. Smith, $\mathrm{PhD}^{\mathrm{c}}$ \\ A. Everette James, JD, MBA ${ }^{\mathrm{d}}$ \\ Brian A. Primack, $\mathrm{MD}, \mathrm{PhD}^{\mathrm{a}}$
}

${ }^{a}$ Center for Research on Media, Technology, and Health, University of Pittsburgh School of Medicine, Pittsburgh, PA

${ }^{\mathrm{b}}$ Pittsburgh Supercomputing Center, Carnegie Mellon University, Pittsburgh, PA

${ }^{\mathrm{c}}$ Department of Computer Science and Engineering, University of Washington, Seattle, WA

${ }^{\mathrm{d}}$ Department of Health Policy and Management, University of Pittsburgh School of Public Health, Pittsburgh, PA

*Corresponding Author:

Jason B. Colditz, MEd

230 McKee Place Suite 600

Pittsburgh, PA 15213

jbc28@ pitt.edu; 412-802-6296 (phone); 412-692-4838 (fax)

Research support: Pennsylvania Department of Health

Acknowledgements: We would like to acknowledge the hard work of the research assistants who were integral in coding and synthesizing new understandings of vaping culture in the "Twittersphere": Mollie March-Steinman, Maharsi Naidu, Kevin Pang, and Christine Stanley. We would also like to acknowledge the editorial assistance of Michelle Woods. 


\begin{abstract}
Few studies have demonstrated the use of mixed methods research to contextualize health topics using primary data from social media. To address this gap in the methodological literature, we present research about electronic nicotine delivery systems (ENDS), using Twitter data from "World Vaping Day." To engage with the quantitative breadth and qualitative depth of 5,149 collected tweets, we utilized a convergent parallel mixed methods framework, integrating thematic prevalence estimates with phenomenological contextualization. Sentiment was more positive than negative across all categories except policy-related. Twenty-three percent of tweets were promotional and relatively few tweets related to tobacco use (4.9\%) or health concerns (4.2\%). Salient themes included modifying or upgrading ENDS devices, and general mistrust of public health advocates and tobacco companies.
\end{abstract}

Keywords: electronic nicotine delivery systems, vaping culture, Twitter, phenomenology, mixed methods 


\section{Introduction}

\section{Mixed Methods Social Media Research}

Mixed methods research approaches have much to offer the study of online social media. Data from social media platforms are both quantitatively large in breadth (i.e., "big data") and qualitatively complex in depth (e.g., unstructured text, linguistically and culturally nuanced). With respect to breadth, mixed methods researchers have posited that content derived from social media is, in some cases, comprehensive enough to be considered population-level data (Mertens et al., 2016). This raises methodological questions about how to appropriately sub-sample the data for feasibility of analyses, how to quantitatively verify the representativeness of a sample within the population, and what qualitative methods are appropriate to incorporate into such frameworks. Snelson (2016) reviewed the literature and identified 55 mixed methods studies relating to social media, published from 2007 through 2013. Of studies that focused on particular social media platforms, those emphasizing Facebook were more prevalent than those related to Twitter or YouTube combined. Methodological approaches generally incorporated two or more primary data sources such as interviews and surveys, as opposed to using multiple methods to approach primary data from social media platforms. Recent studies focusing on primary data from social media platforms tend to implement computational linguistic approaches such as Natural Language Processing (Ruths \& Pfeffer, 2014). While effective for quickly categorizing a large breadth of data, computational linguistic approaches may not effectively synthesize the depth of lived experience from social media content. Yet, such approaches can be integrated with 
qualitative syntheses, within mixed methods frameworks, in order to capture both breadth and depth of meaning from primary social media data. For example, Chou, Prestin, and Kunath (2014) studied obesity across various social media platforms using Natural Language Processing for quantitative classification of themes and discourse analysis to generate illustrative qualitative examples. In mixed methods research, it is additionally useful to describe how such quantitative and qualitative methods are integrated for "added value" over the sum of individual methods themselves (Fetters \& Freshwater, 2015). To this end, Venkatesh, Brown, and Bala (2013) have presented a framework which can help to guide such integrations toward "meta-inferences" for information systems research, but this is not specifically attuned to analysis of primary social media data or health-related content. To date, few studies have demonstrated effective integration of combined quantitative and qualitative approaches to contextualize health topics using primary data from social media.

\section{Twitter and Vaping Culture}

The Twitter social media platform provides an opportunity to observe consistently-sized units of salient content; Twitter messages, known as "tweets," are a maximum of 140 characters. Twitter is also a "public-facing" platform, with an estimated $88 \%$ of users allowing their content to be viewed publicly (Beevolve Inc., 2014). Researchers have used public Twitter data to examine content of messages about topics such as quitting smoking (Prochaska, Pechmann, Kim, \& Leonhardt, 2012) and electronic cigarette marketing (Huang, Kornfield, Szczypka, \& Emery, 2014). Twitter use is highest among adolescents and young adults (Duggan \& Brenner, 2013; Kim et al., 2013), which are populations that have high and increasing incidence of ENDS use 
(Arrazola et al., 2015; Chapman \& Wu, 2014). Twitter users, and those who are also ENDS users in particular, are regularly exposed to ENDS marketing messages on this platform (Emery, Vera, Huang, \& Szczypka, 2014). Analysis of Twitter content may be particularly valuable in exploring cultural contexts of ENDS use (Clark et al., 2014) - a phenomenon which is more broadly referred to as "vaping culture" (Budney, Sargent, \& Lee, 2015; Gostin \& Glasner, 2014).

A detailed investigation into vaping culture is timely given ongoing controversy over the public health impact of ENDS use and regulation (Caponnetto, Saitta, Sweanor, \& Polosa, 2015). For example, ENDS may be used as an approach to quit or cut down on cigarette use (Adriaens, Van Gucht, Declerck, \& Baeyens, 2014; Polosa, Caponnetto, Maglia, Morjaria, \& Russo, 2014), or may have the unintended consequence of leading non-smokers to transition from ENDS use to smoking (Primack, Soneji, Stoolmiller, Fine, \& Sargent, 2015). There are also concerns over toxicants found in ENDS vapor itself (Farsalinos \& Polosa, 2014). While research into health implications of ENDS use remains a high public health priority, additional research is needed to contextualize vaping culture more broadly. For example, Yule and Tinson (2016) organized ENDS users into cultural groups based on various consumer beliefs and behaviors. Their study involved qualitative interviews with a small sample of ENDS users. For future research, they recommended investigating larger samples of ENDS users to elicit broader understandings of vaping culture. Understanding vaping culture, particularly with respect to health beliefs and policy attitudes, may provide insights into how to effectively engage the public about this trend. For example, public health advocates may risk alienating ENDS users if intervention efforts are perceived as ill-informed, overly restrictive, or paternalistic. Collecting relevant data from the Twitter platform offers an opportunity to observe open expression of vaping culture and to 
ultimately gain better understandings of health beliefs, policy attitudes, and other potentially salient aspects of this phenomenon.

\section{Phenomenology to Contextualize Vaping Culture}

In general, phenomenology is a powerful approach to control for bias, as the practice is firmly rooted in traditions of philosophical inquiry and requires researchers to actively monitor the extent to which their subjective experiences accurately reflect objective reality (Yüksel \& Y1ldirım, 2015). That is, phenomenological approaches balance an ontological imperative to recognize the objective existence of phenomena, with an epistemological understanding that actors and observers interpret phenomena through subjective lenses (Cohen \& Omrey, 1994). For this reason, we focused on the ontology of vaping culture and sought to systematically mitigate the subjectivity of both the observers and the observed in order to approach a more “essential” understanding of this phenomenon (Moustakas, 1994).

Recent studies have employed phenomenological approaches to contextualize narratives about social media participation (Rossolatos, 2013), social media protest cultures (Markham, 2014), and Facebook users' experience (Ferrucci \& Tandoc, 2015). To date, the studies at the crossroads of social media use and phenomenology have largely relied on interview data, not taking advantage of the richness present in naturalistic primary social media data. Indeed, phenomenology would not be an appropriate approach for many studies using primary social media data, as the process requires a narrow scope and relatively homogenous sample (Yüksel \& Y1ldırım, 2015). In the present study, we maintained a narrowed scope by targeting ENDS- 
related content obtained from the Twitter platform on the 3rd annual "World Vaping Day" (The Consumer Advocates for Smoke-Free Alternatives Association, 2014). The individual tweets were treated as the formative units for phenomenological synthesis.

The phenomenological process of moving from small units to broader understanding involves "horizonalizing," which relies on valuing each statement or piece of information equally by first breaking them into units of meaning, or "horizons" (Leech \& Onwuegbuzie, 2008). Twitter data are well-suited for this type of approach, as the brevity of individual tweets limits the amount of novel information that each tweet might convey. As such, tweets are already horizonalized to a large extent. Horizons are then grouped into themes and organized into coherent descriptions of phenomena (Moustakas, 1994, p. 97). This differs from qualitative approaches such as discourse analysis where interactions, rather than horizons, are the formative components of synthesis (Starks \& Brown Trinidad, 2007).

When approached thoughtfully, phenomenology is quite compatible with mixed methods approaches (Mayoh \& Onwuegbuzie, 2015). However, the implementation of phenomenological research at-large can seem prohibitively dense with philosophical theory to those unfamiliar with the concept (Caelli, 2001). Thus, a primary goal of the present study was to contribute to the realm of methodological literature: describing processes employed to integrate phenomenological synthesis within a mixed methods study of primary social media data. 


\section{Methodological and Integration Rationale}

In the present study, we developed a mixed-methods approach that allowed us to draw upon both the quantitative breadth as well as the qualitative depth of Twitter data. In approaching these data, we first sought to develop a broad codebook focusing on themes that were particularly relevant to public health and ENDS proliferation. We also focused on positive and negative sentiment toward ENDS among and within thematic categories. The purposive, theoretical structure of our coding framework allowed us to assess the prevalence of conceptually meaningful themes, which may not have organically emerged through use of an inductive coding approach such as grounded theory (Cho \& Lee, 2014; Green, 2014). While coding the data, we also collected memos and observations about emergent themes and examples that did not fit within the defined conceptual framework. In this way, we integrated parallel data analysis and synthesis processes, resulting in both structured data (tweets coded within quantified categories) and unstructured data (coder notes and observations, narratives about emergent themes, demonstrative examples). Integration of a qualitative component was seen as an important step to offset the biases inherent to us, as health science researchers, as we imposed regimented coding frameworks on a complex and nuanced cultural phenomenon.

We synthesized coders' notes and observations within a phenomenological research framework. Such frameworks are often applied in synthesis of interview transcripts, where the researchers benefit from formally acknowledging their preconceptions and biases in order to "bracket out" their subjectivity from the latent phenomena under investigation (Klein \& Westcott, 1994). Our subjectivity was mitigated through iterative processes of synthesizing notes and observations into 
cohesive narratives, pursuing didactic inquiry among coders, developing shared understandings of the interplay among apparent themes, and identifying salient characteristics of vaping culture. Qualitative synthesis occurred throughout the initial codebook development and coding processes, immersed observers in the available cultural discourse, and led to the generation of ancillary content in the form of notes, examples, and narratives. This provided phenomenological context that could be integrated both within and beyond the framework of previously conceptualized categories. In the context of synthesizing and presenting our results, this integration allowed us to (1) add narrative depth around well-defined contextual categories that were quantified in the breadth of our categorical data and (2) explore nuance that was not captured among our preconceived categorical codes.

Another important consideration in mixed methods research is demonstrating the validity of quantitative findings as well as the credibility of qualitative findings (Mertens, 2011). As the previous steps of coding and synthesizing were based on a sub-sample of collected tweets, we enhanced the validity and credibility of findings by comparing these data to the full dataset of collected tweets. We then compared keyword prevalence characteristics (using keywords related to the content area and also popular "hashtags"). In particular, we wanted to be cautious about the potential to over-emphasize the salience of themes that might have been artifacts of researchers' predications toward health-centric thinking. This is also consistent with the step of performing a "validity check" to temper phenomenological interpretation before moving on to presenting a final composite summary (Groenewald, 2004). 
Finally, we integrated the results from these approaches into a comprehensive narrative and discussion. This integrated format provides a well-organized summary for interested practitioners and an example of how seemingly divergent methods can lead to a blended, topical narrative of findings. Thus, the overall goal of our work was two-fold: (1) contributing new understandings about vaping culture that are relevant to public health and (2) providing a novel approach for researchers to integrate phenomenology into a mixed-methods framework for engaging with Twitter data. 


\section{Methods}

\section{Design}

Twitter data are well-suited for a mixed-methods approach because (1) the datasets can be extremely large, lending well to quantitative analyses and (2) the textual content itself can be highly unstructured, which is appropriate for qualitative syntheses. As such, we utilized a “convergent parallel approach" in order to integrate findings from distinct, concurrent analytical approaches toward a holistic understanding of the phenomena under investigation (Cresswell, 2013). This integrative design allowed us to maintain fidelity and separation among one sequence of mixed methods processes which resulted in quantitative findings (See top half of Figure 1) and one qualitative sequence that provided phenomenological context around the data (See bottom half of Figure 1). Findings from these two methodological sequences were then unified and reevaluated through an iterative validation process. The resulting output reflects a "whole through integration that is greater than the sum of the individual qualitative and quantitative parts" and leads toward results that are reported in a thematically united rather than methodologically segregated manner (Fetters \& Freshwater, 2015).

\section{<INSERT FIGURE 1 ABOUT HERE>}

\section{Data Collection}

Accessing and formatting Twitter data. We utilized the Python(x,y) software (2014) to write a custom data collection script built on the Twython package (McGrath, 2014). This 
allowed for reliable access to Twitter's Public Streams API (Twitter Inc., 2014) to retrieve a live feed of public Twitter content. Data were decoded from Twitter's native format and recorded in a structured text file. Recorded data included the timestamp, the textual content of the tweet (images omitted), and the user's Twitter handle (screen name) which was censored prior to coding. This preserved the richness of textual content, while ensuring anonymity of the observed Twitter users, as approved by the University of Pittsburgh Institutional Review Board (IRB \# PRO14070505).

Context of data. We collected data from noon through midnight GMT-5 (U.S. Eastern Standard Time), on September 18, 2014. This date was announced as the 3rd Annual World Vaping Day by the Consumer Advocates for Smoke-Free Alternatives Association (CASAA; 2014), and promoted on websites such as e-cigarette-forum.com, world-vaping-day.com, and vapingday.com. As we were interested in obtaining a more in-depth understanding of vaping culture, focusing data collection on this particular event was ideal. For feasibility of coding, we limited our data to include only tweets that Twitter categorized as written in the English language. The resulting dataset included all tweets meeting this criterion, which contained one or more of the search terms listed below.

Search terms. Previous research of ENDS on Twitter focused primarily on terms such as electronic cigarettes, purposely excluding terms such as vape (Huang et al., 2014). Thus, focusing on World Vaping Day also provided us the opportunity to explore more colloquial search terms characterizing ENDS use. Search keywords used to filter the Twitter stream included vapor, vape, vaping, vapin, vapes, vaper, vapers, vapeon, vapecommunity, ecig, and 
ehookah. This yielded a total of 5,149 tweets which comprised the primary dataset. We engaged with these data using a variety of distinct but interconnected methodological approaches.

\section{Codebook Development}

Based on our interest in synthesizing data that are useful in assessing contexts of public health, policy, and popularity, we framed three broad, a priori coding categories: health-related, policyrelated, and consumer market-related. To further contextualize the data, we also included crosscutting codes for positive or negative sentiment toward ENDS. These codes were dichotomous and independent of one another so that content could be coded as positive, negative, both, or neither. We then refined the codebook through an iterative process of independent coding, collaborative discussion, and code clarification. Two independent coders each assessed 100 randomly selected tweets with the existing codebook, noting areas in which codes might be clarified, split into sub-codes, or combined. They then met with one another and supervising researchers to refine the codebook for future coding. After five iterations of this process, we finalized a codebook that included clear definitions, specific criteria, and exemplar tweets meeting these criteria (Table 1).

This top-down approach was determined to be favorable over a grounded approach in developing the coding framework. It allowed us to assess the prevalence and characteristics of theoreticallyimportant constructs that may not have organically emerged from the data. Throughout codebook development, researchers noted other observations that were not necessarily within the 
scope of established codes. These took the form of notes and memos that were later incorporated into phenomenological synthesis.

\section{<INSERT TABLE 1 ABOUT HERE >}

\section{Quantitative Content Analysis}

Data reduction. To balance coding feasibility and representativeness of content, our quantitative analysis sample was set at 1,000 randomly-selected tweets. Two independent raters who were not involved in initial codebook development double-coded tweets for relevance to ENDS. Tweets were randomized and 200 were coded, inter-rater agreement was examined using Cohen's Kappa statistic ( $\kappa$; Cohen, 1960), and then coding disagreements were collaboratively adjudicated. A second set of 200 tweets was similarly double-coded and adjudicated. Cohen's $\kappa$ increased from .74 (good) to .90 (excellent) by the second set, so the remaining randomized tweets were split evenly and single-coded for relevance to ENDS. We excluded content unrelated to vaping. Because we wished to focus on the use of nicotine, we also excluded content that clearly focused on vaping other types of substances such as cannabis oil. We retained 768 relevant tweets for content coding.

Content coding. Similar to the process used for coding relevance, tweets were independently double-coded in $20 \%$ increments (i.e., $n=154$ out of 768 ). Then, inter-rater agreement was assessed and discrepancies were collaboratively adjudicated in the presence of a supervising researcher. After two rounds coding in this manner, inter-rater agreement was 
sufficient to continue with individual coding (Table 2). In addition to Cohen's $\kappa$, Gwet's $A C_{l}$ was also calculated to assess inter-rater agreement, as this statistic is more robust for lowprevalence content (Gwet, 2008). To examine prevalence and overlap of particular codes within the sample of tweets, we calculated basic descriptive statistics (frequency, percentages, and cross-tabulations) from the resulting data (Table 2). Statistics were calculated using the $\mathrm{R}$ software platform (2015), and inter-rater reliability statistics utilized Gwet's agree.coeff2.r function (2010).

\section{<INSERT TABLE 2 ABOUT HERE>}

\section{Phenomenological Synthesis}

The phenomenological process involves an epistemological focus on identifying and "bracketing out" researcher subjectivity and preconceptions, while working from small units of meaning to a broader understanding and summary (Groenewald, 2004). In our study, bracketing included formally acknowledging the constraints of deductive coding categories and assumptions that accompanied them. For example, coders were apt to perceive ENDS use through lenses of health, policy, and consumer marketing. These coding schemas were important for coding and quantifying within this study and certainly reflected part of vaping culture. However, these schemas functioned at the expense of myriad other novel aspects of vaping culture, which were expressed through individual signals and contexts within the data. 
The primary data for this process were derived from (1) the subset of 500 tweets that were evaluated during codebook development and (2) the subset of 1,000 tweets that were categorically coded. It is important to note that while "synthesis" is explained as a distinct process here, the phenomenological approach was interwoven with the processes of codebook development where coders identified salient themes in the tweets and recorded notes and observations around them. In this way, coders moved from individual phenomenological horizons (i.e., tweets) toward broader themes. Likewise, as coders categorized tweets, they continued to identify nascent themes from tweets that were outside of the established coding framework. Notes and reflections included perspectives of four coders who were well-immersed in the data, as well as supervising researchers who guided coders in identifying possible subjectivity in their observations.

To synthesize these data and observations into broader understandings, coders reviewed their notes and consolidated subjective observations into 1-3 page thematic narratives focusing on what they found to be the most salient and integral concepts for understanding the experience of vaping culture. The narratives were refined during didactic meetings with the larger research team. Meetings focused on understanding convergent and divergent observations and developing a unified and objective understanding of the underlying experience. This process gave voice to both the researched (i.e., Twitter users) and the coders, allowing coders to differentiate their voices from those of the Twitter users. The results of this synthesis took the form of a thematic narrative that was integrated into and around the quantitative results to contextualize the experience of vaping culture on this particular day. By integrating the results of our phenomenological synthesis into the categorical results narrative, we were able to move beyond 
simply reporting percentages and keywords to expound on deeper themes that helped to characterize vaping culture at large.

\section{Validation}

We developed a validation process to enrich "illustration, convergent validation, and analytic density" within our novel mixed-methods approach (Fielding, 2012). This is also consistent with the step of performing a "validity check" to temper phenomenological interpretation, before presenting a final composite summary (Groenewald, 2004). Guided by our previous processes, we revisited the larger data set $(N=5,149)$, to examine prevalence of textual characteristics that related to concepts identified earlier. This provided an additional methodological angle, allowing us to assess the representativeness of the coded subsample and to temper conjectures arising from phenomenological synthesis. To break the text down into component parts, we utilized Python's Twokenize package (O’Connor, Krieger, \& Ahn, 2010; Ott, 2013), which parses Twitter data into individual "tokens" (e.g., words, punctuation, symbols). We then developed a simple script in the Python programming language to quantify the prevalence of tweets that contained particular tokens.

We first searched for the most prevalent hashtags, which served as markers identifying core themes of individual tweets and indicators of shared interests among groups of users (Zappavigna, 2011). This is consistent with how hashtags are intended as "searchable talk" (Zappavigna, 2015). We used the Spearman rank-order correlation coefficient to examine the association between prevalence of the most representative hashtags in the larger dataset versus 
the reduced data that were coded. This provided a quantitative indicator of sampling validity (i.e., representativeness of our coded subsample).

Finally, we conducted targeted searches for other tokens that were related to categories or themes identified in previous methods. To add additional nuance and clarification to emergent themes from our previous approaches, these findings are interwoven into our results narrative. For example, if coders found a particular theme to be salient in earlier steps, we further investigated how frequently keywords related to that theme appear in the larger data. This allowed us to bolster the credibility and trustworthiness of our phenomenological synthesis. 


\section{Results}

\section{Relevance to ENDS}

Of the 1,000 randomized tweets that were coded, 768 were found to be relevant to ENDS and comprise the sample for the study. For the tweets that were excluded, a few thematic trends emerged. For example, the search term vapor yielded the majority of false positives. One reoccurring tweet featured a video where a recently-extinguished candle was relit by igniting its vapor trail (i.e., smoke), and several were about vapor rub (i.e., medicine). Examining the broader data $(N=5,149)$, of the 1,088 tweets that contained the word vapor, $35 \%$ of them also contained either the word trail $(n=285)$ or $r u b(n=94)$. Using terms such as these as exclusion criteria, we would expect improved precision when identifying relevant content by automated keyword searches.

\section{Sentiment}

Positive sentiment was present in $22.9 \%$ of ENDS-relevant tweets, making it more than three times more prevalent than negative sentiment $(7.2 \%)$ in the coded sample $(n=768)$. No tweets were coded as both positive and negative, although it was possible to do so. Tweets that were neither positively nor negatively valenced tended to be more matter-of-fact than opinionated in nature. For example, a tweet like "Going to the hookah bar on Friday" would not be coded as positive or negative unless it included additional context such as "so excited" or "I have no life", respectively. 
Keywords appearing in positively-valenced tweets included general indicators of positive appraisal such as like, love, great, best, premier, attractive, luxury, sweet, or adorbs (slang for adorable). Other keywords included indicators of mood such as grateful, chilled-out, relaxed, or LOL (acronym for laughing out loud). Several themes of interest emerged from within the positively-valenced tweets. For example, we observed enthusiasm surrounding mods and modding ENDS devices, which involves modifying, customizing, and/or upgrading them. We also discovered novel flavors of e-liquid or vape-juice. In this manner, vaping culture seemed to encourage a novel type of experimentation — not only in the traditional sense of trying new substances_-but also in the sense of refining new electronic, mechanical, and aesthetic approaches to create a unique or superior user experience. Related to the novelty of these devices is that they can eschew the stigma that surrounds cigarette smoking, presenting a new opportunity for social bonding. For example, one tweet remarks "Passing a vape pen around the fam dinner table, lmao" ("Imao" is an acronym for laughing my ass off); several other tweets also mentioned sharing ENDS with parents. However, when examining our larger set of tweets - when the terms mom, dad, or parents occurred (41 / 5,149 tweets)—permissiveness was tempered with reflections on parents' disapproval of children using ENDS and/or confiscation of their children's ENDS devices. Thus, while permissiveness was a salient theme within the coded content, validation against the larger dataset demonstrated a broader continuum and refined our understandings of parental reactions to children's ENDS use.

Negative sentiment was best defined by keywords such as hate, stupid, don't like, dumb, loser, lame, nonsense, toxic, despise, dork, or trash. Themes within this coding category included a 
critical attitude towards ENDS itself (e.g., unhealthy, addictive) and more frequently towards ENDS users (e.g., stereotyping users as attempting to look cool or being cliché). Interestingly, sentiments that characterized ENDS as being popular, trendy, or cool tended to be negatively valenced, with the popularity of ENDS being mentioned critically or ironically. For example, one tweet was: "You have an ecig but yet u dont smoke Why in the hell would you buy 1 What a waste of money And how STUPID Yes smoking makes u look cool NOT." Another stated, "Wow you're so cool because you can vape in class can I just be you?" This type of negative valence was common among all tweets where the word cool was used $(68 / 5,149)$, with the exception of a few that used it in a commercial context (e.g., Everyone check out a very cool new custom juice supplier based in Western MA! \#vape \#delish \#ecig \#ejuice).

\section{Commercial Contexts}

Promotional tweets, the most prevalent typology among ENDS-related tweets (23.2\%), were typified by containing brand references or marketing messages that might be further characterized as either a sale or a giveaway. In addition to sale, the former sub-category of tweets often included words like cheap, deal, clearance, or hashtags like \#Discount, \#PromoCode, or \#VoucherCode. Giveaways included words and phrases such as free, sample, raffle, sweepstakes, enter to win, or language to encourage providing non-monetary exchange (e.g., sharing the promotion via social media, taking a survey, or providing contact information). Discounts found in our sample of tweets ranged from 5 to $40 \%$ off the original price. Many tweets also emphasized the urgency of the promotion (i.e., limited time offer, while supplies last). More than a quarter of promotional tweets were of positive sentiment (Table 2), though the 
majority had no detectible sentiment. The few that were of negative sentiment were generally critical of ENDS marketing itself (e.g., "I will NOT miss these blu e-cig commercials!!!! HATE with a passion!!").

Tweets about procurement, obtaining or planning to obtain ENDS-related products, were rarer than promotional content in this sample (3.9\% of coded tweets). In rare cases, the procurement was on behalf of another person (e.g., "Buying my mommy a vape pen like mine but green"). Similarly rare, though related to this theme, was someone losing an ENDS device (e.g., a parent confiscating a child's device).

\section{Health-Related Contexts}

Actual ENDS use was apparent in $6.3 \%$ of relevant tweets. This included cases where individuals mentioned current use or imminent intent to use. Tweets about ENDS use were 10 times as likely to be positive than negative (Table 2), with only two tweets to illustrate negative experiences of ENDS users: "Vape is killing my throat $r n$ " (" $r n$ " is an acronym for "right now") and "Time for a new wick... my ecig tastes like a burnt match." Positive contexts of ENDS use were typified by favorable appraisals of flavorings and ENDS users engaging in other pleasurable activities while using (e.g., "Vapin, drinkin and movies. life is good.").

ENDS-related tweets were associated with tobacco use and by general health in $4.9 \%$ and $4.2 \%$ of relevant tweets, respectively. Sentiment was more positive than negative within these two categories (Table 2), and there was substantial contextual overlap ( $n=22$ with both general 
health and tobacco use coded; $n=26$ with only one of these categories). In cases where both categories were coded, ENDS use was typically expressed as a healthier alternative to tobacco use and as an aid to stop smoking cigarettes. However, when ENDS were not available, users tended to express frustration about using cigarettes instead (e.g., "shouldn't have gone without an ecig because the volume of cigarettes I've been smoking isn't helping anything"). A sub-context of note is the availability of organic ENDS liquid, promoted as a healthier alternative to contemporary varieties (e.g., "Use only Organic E-liquid if you getting sick from VP Juice"). In the larger data set, the term organic appeared only in 4 ENDS-relevant tweets, but it appeared in 21 irrelevant tweets in relation to organic vapor cartridge (i.e., respirator equipment). This presents an additional phrase to exclude when automating searches.

General health was coded in 33 tweets, with 13 of those occurrences being the same tweet: "\#ecigs It is not the problem of vapers if "public health" people suffer from cognitive dissonance. Listen, learn!" Several of the other health-related tweets expressed a similar mistrust of "public health grandees" $(n=11)$. The sense that public health officials and researchers are at odds with vaping culture was a recurring theme. 


\section{Legal and Policy Contexts}

Within the sample, only $3.5 \%$ of tweets included references to legal policy or regulation. Fewer than $15 \%$ of these tweets had any detectable sentiment; these were typically reports from news outlets about states or municipalities placing restrictions on ENDS use. The more sentimental tweets originated from ENDS advocates (e.g., "any vote to stifle \#ecig sales through taxation is a vote EMBRACING combustible cigarettes" and "Vuze \& Blu want to ban ecigs, can we stop saying Big T'own' ecigs now and admit that regulation will hand it 2 them"). The emergent concern in vaping culture appeared to be on "big tobacco" entering the market and vying for regulatory restrictions on ENDS. The large electronic cigarette brands were generally portrayed in an unfavorable context, as they are seen as trying to force smaller companies and refillable devices out of the market. Many of the 38 mentions of Vuse or Blu (which are affiliated with tobacco companies) expressed concern over tobacco company influence on the ENDS market. For example, tweets relevant to these brands expressed: anger about companies lobbying to restrict ENDS availability or functionality $(n=7)$, frustration about brands' television advertisements $(n=3)$, or displeasure toward a particular marketing representative $(n=2)$. An additional 25 tweets related to Vuse and Blu involved third-parties selling device carrying cases.

\section{World Vaping Day and Associated Hashtags}

Our perspectives on sentiment, marketing, health, and policy are different angles to understand World Vaping Day and broader vaping culture. Within a phenomenological lens, both of these phenomena are far more nuanced than categorical descriptions capture. For example, the 
\#WorldVapingDay hashtag spanned multiple contexts of product promotion, health, and policy. However, there were only 9 occurrences in the coded data and 44 in the larger dataset. While this frequency is nearly 10 times higher than the number of instances that an average hashtag appeared in the larger data $($ mean $=4.6$, median $=1)$, it paled in comparison to other popular hashtags represented (Table 3). Thus, it was not clear whether many of the people who were tweeting about ENDS on this particular day were aware of the World Vaping Day phenomenon. Overall, it appeared to function as a platform to market ENDS devices, oppose ENDS regulation, and offer congratulations to individuals who transitioned from smoking to vaping (e.g., "Happy \#WorldVapingDay! Congrats to all of those who have made the switch to the adult alternative! \#vapor \#ecig"). In short, World Vaping Day meant different things to different people, though perhaps not to many people overall.

\section{<INSERT TABLE 3 ABOUT HERE>}

Given the paucity of content dedicated to World Vaping Day, as compared to the larger amount of more general ENDS-related data, our aforementioned results may better reflect a slightly broader scope of understanding vaping culture. Thus, while individuals may have been increasingly vocal or active on that particular day, the messages reflect back to vaping culture at large. Hashtags that help to identify that culture included \#vapelife (also \#vapelyfe) and \#vapecommunity (Table 3). In contrast to \#worldvapingday spanning multiple contexts within our coded data, neither \#vapelife nor \#vapelyfe hashtags appeared in health- or policy-related tweets. These hashtags were also slightly more prevalent within the category of promotional tweets (9.0\%) as compared to within non-promotional tweets (5.6\%). The \#vapecommunity 
hashtag, while less prevalent overall, was more prevalent within non-promotional tweets $(2.4 \%$ vs. 1.9\%). This hashtag was also present within two health-related tweets and one policy-related tweet. Thematically, the \#vapecommunity hashtag seemed to be more aligned with \#worldvapingday, whereas \#vapelife variants seemed to be more closely tied to commercialization and portraying a cool or edgy image. The differing themes among \#worldvapingday, \#vapelife and \#vapelyfe, and \#vapecommunity are important in understanding how similar hashtags may exhibit different cultural values within groups such as ENDS users.

\section{Uncategorized or Cross-Cutting Themes}

As reflected in Table 2, 59\% of the relevant data were not categorized into any of our thematic coding categories. Some of the emergent themes from these data were, however, reflected within results reported earlier in this paper. For example, aspects of coolness, un-coolness, and irony were reflected within sentiment. Similarly, we discovered cross-cutting themes of approval vs. disapproval, and parents' leniency vs. sternness. We also discovered contexts of technical expertise (e.g., modding), discerning taste (e.g., best flavors), and special techniques (e.g., blowing rings or big clouds).

There was also content that, while relevant to ENDS-use, was more about the social networking in general. For example, there were messages that utilized ENDS as a means for building online social capital, such as "Follow if you \#vape" and "Thanks for the follow! \#vapeon." Other messages included general observations and humor surrounding ENDS use, such as "Girl at cherry berry *bite of ice cream* *hit ecig* *repeat*" and "So where's the vape pencil?" 


\section{Discussion}

ENDS use, commonly called "vaping," is growing in popularity and presents health risks related to inhalation of toxic chemicals as well as serving as a gateway to cigarette use among nonsmokers (Farsalinos \& Polosa, 2014; Leventhal et al., 2015; Primack et al., 2015). Youth are increasingly using ENDS products (Arrazola et al., 2015), and there are concerns that this trend may relate to the perceived coolness or allure of "vaping culture" (Budney et al., 2015; Gostin \& Glasner, 2014). Our study extends existing research that has utilized public data from the Twitter social media platform to examine smoking and electronic cigarette trends (Huang et al., 2014; Myslin et al., 2013). In this study, we focused specifically on popular vaping culture by monitoring relevant Twitter content from World Vaping Day 2014. Using a mixed-methods approach, we were able to capitalize on both the breadth and depth inherent to Twitter data. Our approach allowed us to contextualize sentiment toward ENDS and to discover new insights to inform public health research and practice. Our results indicate that vaping culture is multifaceted across commercial, health, and policy contexts. Within each of these contexts, there was a dynamic interplay of perspectives that help to better understand vaping culture.

With respect to commercial-related content, we found only $23 \%$ of the ENDS-related tweets to be promotional in nature, with an additional $4 \%$ about procuring ENDS. However, a recent cross-sectional study by Huang et al. (2014) found that approximately $90 \%$ of collected tweets about electronic cigarettes were commercial in nature. Some of this difference may be due to omitting electronic cigarette brand names from our search strings and utilizing different coding criteria for commercial content. However, some of the difference may also be due to the 
evolving commercial landscape of ENDS. The vaping culture that we observed appeared to have a less favorable view of older electronic cigarette products as compared to newer devices that are upgradable, customizable, and refillable with various flavors of e-liquid. This is consistent with qualitative findings on the high desirability of these newer ENDS features (Cooper, Harrell, \& Perry, 2016). This was further manifested by criticisms of "big tobacco" companies that encourage regulation of ENDS devices while also being affiliated with dominant brands of contemporary electronic cigarettes. Taken together with vaping culture's critical lens on individuals who presumably use ENDS to appear "cool," this points toward a more nuanced cultural understanding of ENDS commercialization.

Consistent with conceptual work in marketing research (Nancarrow, Nancarrow, \& Page, 2002), attitudes toward ENDS' "coolness" reflected aspects of authenticity and membership in socially exclusive groups. For example, "modders" are individuals capable of modifying, upgrading, and customizing ENDS devices. Additionally, some individuals presented themselves as connoisseurs or opinion leaders with respect to e-liquid flavors. More casual use of ENDS, and especially in school settings or by people who were not using ENDS to quit smoking was sometimes perceived as inauthentic (i.e., un-cool). Future research and health promotion advocacy related to ENDS might benefit from considering these aspects of perceived authenticity for ENDS users. It may also be useful to further examine specific sub-groups such as modders, $e$ liquid enthusiasts, or ex-smokers as "vaping subcultures" with distinct types of knowledge, beliefs, and behaviors. Additionally, focusing on device components, configurations, and eliquid varieties will provide new insights into trends surrounding novel ENDS devices that may be gaining popularity. 
Health-related themes were largely dominated by messages about harm reduction for smoking cessation or the relative safety of ENDS use. At one extreme were messages such as "it's just water vapor" and at the other were messages such as "that can't be good for you." Despite searching for relevant keywords in the data, we were unable to locate content that was wellinformed by specific health risks of ENDS use. This is consistent with recent qualitative research where current and former smokers demonstrated conflicting health beliefs and a dearth of concrete knowledge about possible ENDS health effects (Rooke, Cunningham-Burley, \& Amos, 2016). While previous research has demonstrated potential risks of particular chemicals in eliquids (Farsalinos \& Polosa, 2014) and likelihood of ENDS users to engage in cigarette smoking (Leventhal et al., 2015; Primack et al., 2015), these public health messages seemed to be largely omitted from our data. There are also potential benefits of ENDS use for harm reduction and smoking cessation (Adriaens, Van Gucht, Declerck, \& Baeyens, 2014; Polosa, Caponnetto, Maglia, Morjaria, \& Russo, 2014). These messages, while clearly present in vaping culture, were somewhat overshadowed by commercial overtones and novel facets of ENDS use such as customizability and flavors. Overall, there was a lack of evidence-based health information in the public Twitter sphere. This represents a possible opportunity for public health advocates to enter into dialogue about potential ENDS benefits and risks. It may be beneficial for public health education campaigns to enter the dialogue and allocate resources for disseminating pertinent information through the Twitter platform. However, it is also important to consider how messages of this nature might be received by current and potential ENDS users. In our data, we observed mistrust toward public health advocates who were understood to be misaligned with the harm-reduction benefits surrounding ENDS use. Thus, it may be valuable for public health 
advocates to provide information about known risks while clearly empathizing with the perceived harm-reduction benefits of ENDS in smoking cessation. Additional knowledge of specific e-liquid constituents and ENDS use trends will help to frame specific intervention messages that might be well-received by current and potential ENDS users. In our data there were cases where individuals had expressed intention to smoke cigarettes if their ENDS devices were taken away (e.g., as a threat to parents who confiscated the ENDS devices). Additionally, there were cases where individuals regretted that they had smoked cigarettes when their ENDS devices were misplaced or when batteries were depleted. Better understanding this substitution effect may be an important consideration for researchers and health practitioners.

While there are known hurdles to translating research into evidence-based policy reforms (Brownson, Chriqui, \& Stamatakis, 2009), researchers and public health advocates have been actively providing ENDS-related insights and recommendations specifically for policymakers (Brandon et al., 2015; Henningfield \& Zaatari, 2010). These recommendations encourage policymakers to keep pace with the scientific literature, gain a clear understanding of new trends in ENDS devices and use patterns, and acknowledge both the pros and cons of ENDS use. We observed concerns about policymakers making decisions that were either uninformed or misinformed, either by tobacco companies or public health advocates with political agendas. In light of this, it seems that both the public health community and vaping culture encourage informed policy decision making regarding ENDS. Based on themes within our data, however, it is likely that even well-informed regulations will face challenges by members of vaping culture who are opposed to regulation in general. Our observations on ENDS users' aversion to ENDS regulation are consistent with recent survey research on the topic (Wackowski \& Delnevo, 
2015). Based on current understandings, it may be beneficial for health policy advocates in this realm to more clearly distinguish and distance themselves from political interests and industry funding stemming from "big tobacco." Demonstrating that policy recommendations are based in the best available science and clearly acknowledging conflicts of interest (perceived or otherwise) may help to bridge ideological divides with vaping culture. Additional research on patterns of cigarette and ENDS use — as well as ENDS users' health beliefs — will help to further understand these complex relationships within a broader cultural context.

\section{Methodological Significance}

We developed a novel mixed methods approach that capitalized on both the quantitative breadth as well as the qualitative depth of primary Twitter data. This project utilized an integrated process of codebook development, categorical coding, in-depth phenomenological synthesis of content, and validation of emergent understandings. While contemporary phenomenological approaches have focused primarily on qualitative processes, there is an established need to conduct and document mixed methods approaches in this realm (Mayoh \& Onwuegbuzie, 2015).

Our codebook was broadly framed with a priori categories to gain understandings about contexts particularly relevant to public health. We refined the codebook through an iterative process of independent and collaborative coding. Within a random subset of the data, inter-rater reliability was assessed using both Cohen's $\kappa$ and Gwet's $A C_{l}$, and thematic prevalence was quantified. Throughout the codebook development and coding processes, coders organized salient examples of tweets (i.e., phenomenological horizons), made notes, developed broader narratives, and 
shared reflections in didactic sessions. Undertaking this process within a phenomenological research framework provided broad understandings of vaping culture within and beyond established coding categories. Emergent understandings were validated against both broad and targeted keyword searches of the primary data and by quantitatively examining hashtag prevalence between the primary dataset and the coded subset.

Our results were broadly presented within our a priori thematic categories as headings, where appropriate. Under thematic headings, results narratives integrated findings from all methodological aspects of the study. This integration of qualitative and quantitative findings allowed us to present comprehensive and meaningful results within discrete themes and to identify themes that did not fit within our preconceived categorical schema. The integration of multiple methods among thematic categories also served to enhance readability for those interested in particular themes and demonstrated one way of presenting integrated mixed methods research results.

\section{Limitations}

Our data were inclusive of a 12 hour span of live tweets collected on World Vaping Day, and these results may not reflect the broader context of ENDS use on a day-to-day basis. Rather, this study focused on identifying and describing novel contexts of vaping culture that may be useful in broader studies of ENDS use. Using targeted keyword searches and a random subsample of data facilitated a manageable dataset for categorical coding and synthesis. However, this approach did not capture the natural flow of conversations on the Twitter platform. This would 
have been a major impediment to discourse analysis approaches, though it was less problematic for our particular phenomenological approach which relied on individual tweets as formative thematic units (i.e., phenomenological horizons). A longer timeframe of data collection, the inclusion of Twitter metadata (e.g., usernames, locations, social influence, profile characteristics), and including social network analysis frameworks will be beneficial for assessing broader trends and understanding the active conversations that surround this type of social phenomenon on the Twitter platform. This would be a labor-intensive process for human coders, and continued technological innovations may help to streamline and add additional context to this type of data.

\section{Future Directions}

The in-depth, manual coding of this content provides a springboard for follow-up studies that can refine the approaches and build from our coded data using machine learning processes. Using the human-coded data from this study, we can begin to refine search strategies for ENDS-related content in light of word-sense disambiguation (Stevenson \& Wilks, 2003). For example, machine learning algorithms may be able to more efficiently classify content in which the term vapor refers to topics outside the scope of ENDS discussion. Further, these human-coded data can be used to train automatic classifiers to detect ENDS-related sentiment and sub-contexts (e.g., commercial, health-related, policy-related) in greater breadth than would be feasible for human coders. This will further expand our understanding of ENDS, likely discovering trends among ENDS and traditional cigarette use. This may also lead toward understanding other public health topics such as drug and alcohol use which may have similar sub-contexts to evaluate. Using 
automated processes to expand this research to larger data sets will also allow for (1) tracking relevant Twitter accounts over time, (2) investigating social relationships among promoters and ENDS users, and (3) further refining understandings gained through the current study. This will help to refine broader understandings of how Twitter is used to promote and normalize ENDS use, as well as how health advocates can best engage in dialogue around this emergent trend.

\section{Conclusions}

World Vaping Day was primarily characterized by the promotion of ENDS devices and associated parts/accessories for modifying and refilling them (e.g., high-power batteries, unique customizations, novel flavors of e-liquid). Experimenting with new ENDS customizations and eliquid flavors generated some excitement, whereas the tone towards contemporary electronic cigarettes was more critical. Specific health concerns surrounding ENDS use were practically non-existent. ENDS were typically presented favorably as compared to cigarettes, both in terms of healthiness and general social approval (e.g., permissiveness or "coolness"). There was however, a critical tone toward individuals using ENDS to appear cool rather than as a tobacco cessation aide. There was also criticism and mistrust toward both "big tobacco" and public health advocates who are perceived to have vested interests in ENDS regulation. These are trends that researchers and practitioners should be aware of when understanding and engaging in dialogue related to ENDS usage. Continued research and effective translational approaches are needed to inform the public health community of these emerging trends in vaping culture, which will be integral to a broader understanding of ENDS use. 


\section{References}

Adriaens, K., Van Gucht, D., Declerck, P., \& Baeyens, F. (2014). Effectiveness of the electronic cigarette: An eight-week flemish study with six-month follow-up on smoking reduction, craving and experienced benefits and complaints. International Journal of Environmental Research and Public Health, 11(11), 11220-11248. http://doi.org/10.3390/ijerph111111220

Arrazola, R. A., Singh, T., Corey, C. G., Husten, C. G., Neff, L. J., Apelberg, B. J., ... Caraballo, R. S. (2015). Tobacco use among middle and high school students - United States, 20112014. Morbidity and Mortality Weekly Report (Vol. 64). Retrieved from http://www.cdc.gov/mmwr/preview/mmwrhtml/mm6414a3.htm

Beevolve Inc. (2014). An exhaustive study of Twitter users across the world. Retrieved from http://www.beevolve.com/twitter-statistics/

Brandon, T. H., Goniewicz, M. L., Hanna, N. H., Hatsukami, D. K., Herbst, R. S., Hobin, J. A., ... Warren, G. W. (2015). Electronic nicotine delivery systems: A policy statement from the American Association for Cancer Research and the American Society of Clinical Oncology. Journal of Clinical Oncology, 33(8), 952-963. http://doi.org/10.1200/JCO.2014.59.4465

Brownson, R. C., Chriqui, J. F., \& Stamatakis, K. A. (2009, September). Understanding evidence-based public health policy. American Journal of Public Health. http://doi.org/10.2105/AJPH.2008.156224

Budney, A. J., Sargent, J. D., \& Lee, D. C. (2015). Vaping cannabis (marijuana): Parallel concerns to e-cigs? Addiction, 110(11), 1699-1704. http://doi.org/10.1111/add.13036

Burton, S., Dadich, A., \& Soboleva, A. (2013). Competing voices: Marketing and countermarketing alcohol on Twitter. Journal of Nonprofit \& Public Sector Marketing, 25(2), 186209. http://doi.org/10.1080/10495142.2013.787836

Caelli, K. (2001). Engaging with phenomenology: Is it more of a challenge than it needs to be? Qualitative Health Research, 11(2), 273-281. http://doi.org/10.1177/104973201129118993

Caponnetto, P., Saitta, D., Sweanor, D., \& Polosa, R. (2015). What to consider when regulating electronic cigarettes: Pros, cons and unintended consequences. International Journal of Drug Policy, 26(6), 554-559. http://doi.org/10.1016/j.drugpo.2015.03.001

Chapman, S. L. C., \& Wu, L. T. (2014). E-cigarette prevalence and correlates of use among adolescents versus adults: A review and comparison. Journal of Psychiatric Research. http://doi.org/10.1016/j.jpsychires.2014.03.005

Cho, J. Y., \& Lee, E. (2014). Reducing confusion about grounded theory and qualitative content analysis: Similarities and differences. The Qualitative Report, 19(64), 1-20. Retrieved from http://www.nova.edu/ssss/QR/QR19/cho64.pdf

Chou, W. S., Prestin, A., \& Kunath, S. (2014). Obesity in social media: A mixed methods analysis. Translational Behavioral Medicine , 4(3), 314-323. doi:10.1007/s13142-014-02561

Clark, E. M., Jones, C., Gaalema, D., White, T. J., Redner, R., Everett, R., ... Danforth, C. (2014). Social media meets population health: A sentiment and demographic analysis of tobacco and e-cigarette use across the "Twittersphere." Value in Health, 17(7), A603. http://doi.org/10.1016/j.jval.2014.08.2093 
Cohen, J. (1960). A coefficient of agreement for nominal scales. Educational and Psychological Measurement, 20(1), 37-46. http://doi.org/10.1177/001316446002000104

Cohen, M. Z., \& Omery, A. (1994). Schools of phenomenology: Implications for research. Critical Issues in Qualitative Research Methods, 2, 136-153

Cooper, M., Harrell, M. B., \& Perry, C. L. (2016). A qualitative approach to understanding realworld electronic cigarette use: Implications for measurement and regulation. Preventing Chronic Disease, 13, 150502. http://doi.org/10.5888/pcd13.150502

Cresswell, J. W. (2013). Research design: Qualitative, quantitative, and mixed methods approaches. Thousand Oaks, CA: Sage Publications.

Duggan, M., \& Brenner, J. (2013). The demographics of social media users. - 2012. Washington, DC. Retrieved from http://pewinternet.org/Reports/2013/Social-mediausers.aspx

Emery, S. L., Vera, L., Huang, J., \& Szczypka, G. (2014). Wanna know about vaping? Patterns of message exposure, seeking and sharing information about e-cigarettes across media platforms. Tobacco Control, 23 Suppl 3, iii17-25. http://doi.org/10.1136/tobaccocontrol2014-051648

Farsalinos, K. E., \& Polosa, R. (2014). Safety evaluation and risk assessment of electronic cigarettes as tobacco cigarette substitutes: A systematic review. Therapeutic Advances in Drug Safety, 5(2), 67-86. http://doi.org/10.1177/2042098614524430

Ferrucci, P., \& Tandoc, E. C. (2015). The Facebook experience: A phenomenology of Facebook use. Online Journal of Communication and Media Technologies, 5(3), 176-197. Retrieved from http://www.ojcmt.net/articles/53/5311.pdf

Fetters, M. D., \& Freshwater, D. (2015). The $1+1=3$ Integration Challenge. Journal of Mixed Methods Research, 9(2), 115-117. doi:10.1177/1558689815581222

Fielding, N. G. (2012). Triangulation and mixed methods designs: Data integration with new research technologies. Journal of Mixed Methods Research, 6(2), 124-136. http://doi.org/10.1177/1558689812437101

Gostin, L. O., \& Glasner, A. Y. (2014). E-Cigarettes, vaping, and youth. JAMA, 312(6), 595. http://doi.org/10.1001/jama.2014.7883

Green, H. E. (2014). Use of theoretical and conceptual frameworks in qualitative research. Nurse Researcher, 21(6), 34-38. http://doi.org/10.7748/nr.21.6.34.e1252

Groenewald, T. (2004). A phenomenological research design illustrated. International Journal of Qualitative Methods, 3(1), 42-55. http://doi.org/10.1177/160940690400300104

Gwet, K. L. (2008). Computing inter-rater reliability and its variance in the presence of high agreement. British Journal of Mathematical and Statistical Psychology, 61(1), 29-48. http://doi.org/10.1348/000711006X126600

Gwet, K. L. (2010). R functions for calculating agreement coefficients. Gaithersburg, MD: Advanced Analytics, LLC. Retrieved from http://www.agreestat.com/r_functions.html

Henningfield, J. E., \& Zaatari, G. S. (2010). Electronic nicotine delivery systems: Emerging science foundation for policy. Tobacco Control, 19(2), 89-90.

http://doi.org/10.1136/tc.2009.035279 
Huang, J., Kornfield, R., Szczypka, G., \& Emery, S. L. (2014). A cross-sectional examination of marketing of electronic cigarettes on Twitter. Tobacco Control, 23 Suppl 3, iii26-30. http://doi.org/10.1136/tobaccocontrol-2014-051551

Kim, A. E., Hansen, H. M., Murphy, J., Richards, A. K., Duke, J., \& Allen, J. A. (2013). Methodological considerations in analyzing Twitter data. Journal of the National Cancer Institute, 2013(47), 140-146. http://doi.org/10.1093/jncimonographs/lgt026

Klein, P., \& Westcott, M. R. (1994). The changing character of phenomenological psychology. Canadian Psychology, 35(2), 133-158. http://doi.org/10.1037/0708-5591.35.2.133

Leech, N. L., \& Onwuegbuzie, A. J. (2008). Horizonalization. In L. Given (Ed.), The SAGE Encyclopedia of Qualitative Research Methods (Online). 2455 Teller Road, Thousand Oaks California 91320 United States: SAGE Publications, Inc. http://doi.org/10.4135/9781412963909

Leventhal, A. M., Strong, D. R., Kirkpatrick, M. G., Unger, J. B., Sussman, S., Riggs, N. R., ... Audrain-McGovern, J. (2015). Association of electronic cigarette use with initiation of combustible tobacco product smoking in early adolescence. JAMA, 314(7), 700. http://doi.org/10.1001/jama.2015.8950

Markham, T. (2014). Social media, protest cultures and political subjectivities of the Arab spring. Media, Culture \& Society, 36(1), 89-104. http://doi.org/10.1177/0163443713511893

Mayoh, J., \& Onwuegbuzie, A. J. (2015). Toward a conceptualization of mixed methods phenomenological research. Journal of Mixed Methods Research, 9(1), 91-107. http://doi.org/10.1177/1558689813505358

McGrath, R. (2014). Twython. Retrieved from http://pypi.python.org/pypi/twython

Mertens, D. M. (2011). Publishing mixed methods research. Journal of Mixed Methods Research, 5(1), 3-6. http://doi.org/10.1177/1558689810390217

Mertens, D. M., Bazeley, P., Bowleg, L., Fielding, N., Maxwell, J., Molina-Azorin, J. F., \& Niglas, K. (2016). Expanding thinking through a kaleidoscopic look into the future: Implications of the Mixed Methods International Research Associations Task Force Report on the future of mixed methods. Journal of Mixed Methods Research, 10(3), 221-227. doi: $10.1177 / 1558689816649719$

Moustakas, C. (1994). Phenomenological research methods. Thousand Oaks, CA: Sage Publications.

Myslin, M., Zhu, S. H., Chapman, W., \& Conway, M. (2013). Using twitter to examine smoking behavior and perceptions of emerging tobacco products. Journal of Medical Internet Research, 15(8), e174. http://doi.org/10.2196/jmir.2534

Nancarrow, C., Nancarrow, P., \& Page, J. (2002). An analysis of the concept of cool and its marketing implications. Journal of Consumer Behaviour, 1(4), 311-322. http://doi.org/10.1002/cb.77

O’Connor, B., Krieger, M., \& Ahn, D. (2010). TweetMotif : Exploratory search and topic summarization for Twitter. In 4th International AAAI Conference on Weblogs and Social Media (pp. 2-3). Washington, DC. http://doi.org/10.1145/1390334.1390389

Ott, M. (2013). Twokenize (ark-twokenize-py). Retrieved from https://github.com/myleott/ark- 
twokenize-py

Polosa, R., Caponnetto, P., Maglia, M., Morjaria, J. B., \& Russo, C. (2014). Success rates with nicotine personal vaporizers: A prospective 6-month pilot study of smokers not intending to quit. BMC Public Health, 14, 1159. http://doi.org/10.1186/1471-2458-14-1159

Primack, B. A., Soneji, S., Stoolmiller, M., Fine, M. J., \& Sargent, J. D. (2015). Progression to traditional cigarette smoking after electronic cigarette use among US adolescents and young adults. JAMA Pediatrics, 169(11), 1018. http://doi.org/10.1001/jamapediatrics.2015.1742

Prochaska, J. J., Pechmann, C., Kim, R., \& Leonhardt, J. M. (2012). Twitter=quitter? An analysis of Twitter quit smoking social networks. Tobacco Control, 21, 447-449. http://doi.org/10.1016/j.biotechadv.2011.08.021.Secreted

R Core Team. (2014). R: A language and environment for statistical computing. Vienna, Austria: R Foundation for Statistical Computing. Retrieved from http://www.r-project.org/

Rooke, C., Cunningham-Burley, S., \& Amos, A. (2016). Smokers' and ex-smokers' understanding of electronic cigarettes: A qualitative study. Tobacco Control, 25(e1), e60e66. http://doi.org/10.1136/tobaccocontrol-2014-052151

Rossolatos, G. (2013). The brand comments: A social phenomenological/semiotic account of the formation of the (inter)subjective habitus through social media participatory mechanisms. SSRN Electronic Journal. http://doi.org/10.2139/ssrn.2221263

Ruths, D., \& Pfeffer, J. (2014). Social media for large studies of behavior. Science, 346(6213), 1063-1064. doi:10.1126/science.346.6213.1063

Snelson, C. L. (2016). Qualitative and mixed methods social media research: A review of the literature. International Journal of Qualitative Methods, 15(1). doi:10.1177/1609406915624574

Starks, H., \& Brown Trinidad, S. (2007). Choose Your Method: A Comparison of Phenomenology, Discourse Analysis, and Grounded Theory. Qualitative Health Research, 17(10), 1372-1380. doi:10.1177/1049732307307031

Stevenson, M., \& Wilks, Y. (2003). Word-sense disambiguation. In R. Mitkov (Ed.), The Oxford handbook of computational linguistics (pp. 249-265). New York.

The Consumer Advocates for Smoke-Free Alternatives Association. (2014). International call to action! Celebrate World Vaping Day by telling the World Health Organization how ecigarettes have changed your life. Retrieved November 18, 2015, from http://blog.casaa.org/2014/09/international-call-to-action-celebrate.html

Twitter Inc. (2014). Public API. Retrieved from http://dev.twitter.com/streaming/public

Venkatesh, V., Brown, S. A., \& Bala, H. (2013). Bridging the qualitative-quantitative divide: Guidelines for conducting mixed methods research in information systems. Management Information Systems Quarterly, 37(1), 21-54. Retrieved from http://aisel.aisnet.org/misq/vol37/iss1/3/

Wackowski, O. A., \& Delnevo, C. D. (2015). Smokers' attitudes and support for e-cigarette policies and regulation in the USA. Tobacco Control, 24(6), 543-546. http://doi.org/10.1136/tobaccocontrol-2014-051953

Yüksel, P., \& Yıldırım, S. (2015). Theoretical frameworks, methods, and procedures for conducting phenomenological studies. Turkish Online Journal of Qualitative Inquiry, 6(1), 
1-20. http://doi.org/10.17569/tojqi.59813

Yule, J. A., \& Tinson, J. S. (2016). Youth and the sociability of "Vaping." Journal of Consumer Behaviour, [Online be. doi:10.1002/cb.1597

Zappavigna, M. (2011). Ambient affiliation: A linguistic perspective on Twitter. New Media \& Society, 13(5), 788-806. http://doi.org/10.1177/1461444810385097

Zappavigna, M. (2015). Searchable talk: The linguistic functions of hashtags. Social Semiotics, 25(3), 274-291. http://doi.org/10.1080/10350330.2014.996948 
Table 1. Definitions for categorical codes and example tweets.

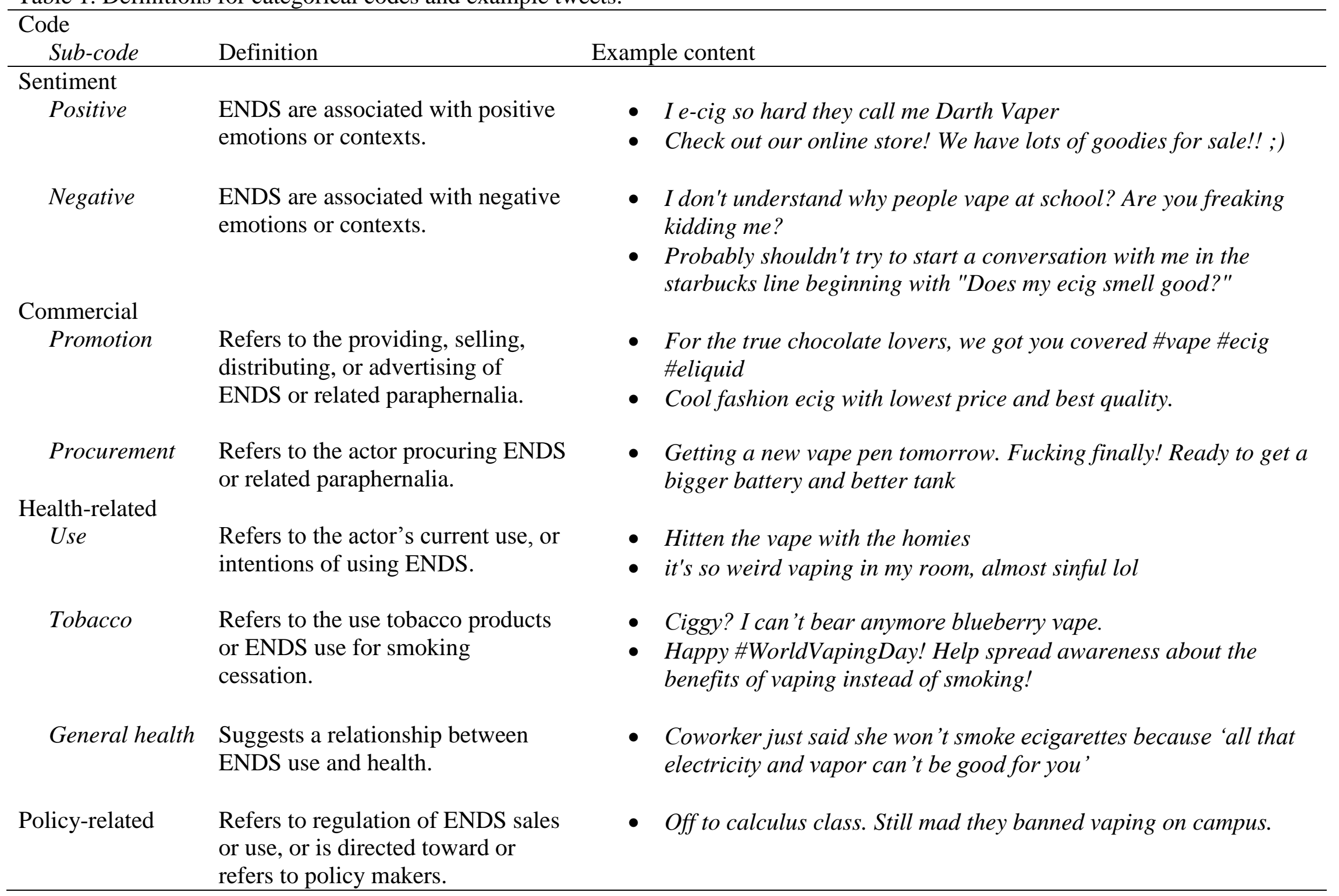


Table 2. Descriptive statistics and sentiment comparison among ENDS contexts $(n=768)$

\begin{tabular}{|c|c|c|c|c|c|}
\hline \multirow[b]{2}{*}{ Context } & \multirow[b]{2}{*}{$n$} & \multicolumn{2}{|c|}{ Sentiment \%* } & \multicolumn{2}{|c|}{ Inter-rater agreement** } \\
\hline & & Positive & Negative & Cohen's $\kappa$ & Gwet's $A C_{l}$ \\
\hline Not categorized & 451 & 18.6 & 10.0 & - & - \\
\hline \multicolumn{6}{|l|}{ Commercial } \\
\hline Promotion & 178 & 25.8 & 0.6 & .70 & .83 \\
\hline Procurement & 30 & 33.3 & 3.3 & .65 & 97 \\
\hline \multicolumn{6}{|l|}{ Health } \\
\hline Use & 48 & 43.8 & 4.2 & .60 & .93 \\
\hline Tobacco & 38 & 39.5 & 10.5 & .84 & .98 \\
\hline General health & 32 & 40.6 & 6.3 & .76 & .97 \\
\hline Policy & 27 & 3.7 & 11.1 & .97 & $>.99$ \\
\hline \multicolumn{6}{|l|}{ Overall sentiment } \\
\hline Positive & 176 & 100 & - & .88 & .91 \\
\hline Negative & 55 & - & 100 & .92 & .99 \\
\hline
\end{tabular}

* Percent values are reported by row.

** Based on a random subset of 154 relevant tweets with two independent raters. 
Table 3. Top 20 Hashtags in the full dataset $(\mathrm{N}=5,149)$ and coded subset of relevant tweets $(n=$ 768).

\begin{tabular}{|c|c|c|}
\hline Hashtag & Full dataset & Coded subset \\
\hline \#vape & 871 & 133 \\
\hline \#ecig & 418 & 56 \\
\hline \#vaping & 283 & 54 \\
\hline \#ecigs & 203 & 40 \\
\hline \#vapelife & 179 & 39 \\
\hline \#vapor & 132 & 14 \\
\hline \#vaporizer & 118 & 16 \\
\hline \#ejuice & 99 & 25 \\
\hline \#vapeon & 97 & 22 \\
\hline \#eliquid & 91 & 17 \\
\hline \#vapecommunity & 75 & 13 \\
\hline \#vapers & 73 & 19 \\
\hline \#reddit & 62 & 11 \\
\hline \#sale & 51 & 9 \\
\hline \#vapelyfe & 51 & 10 \\
\hline \#buy & 49 & 7 \\
\hline \#vapeporn & 43 & 5 \\
\hline \#worldvapingday & 43 & 9 \\
\hline \#checkout & 41 & 8 \\
\hline \#iphone6 ${ }^{\mathrm{a}}$ & 38 & 6 \\
\hline
\end{tabular}

a The \#iphone6 hashtag related to a phone case that was "vapor" colored. Hashtags after the top 20 were decreasingly relevant to ENDS or vaping culture. 
Figure 1. Convergent parallel mixed methods approach, illustrated.

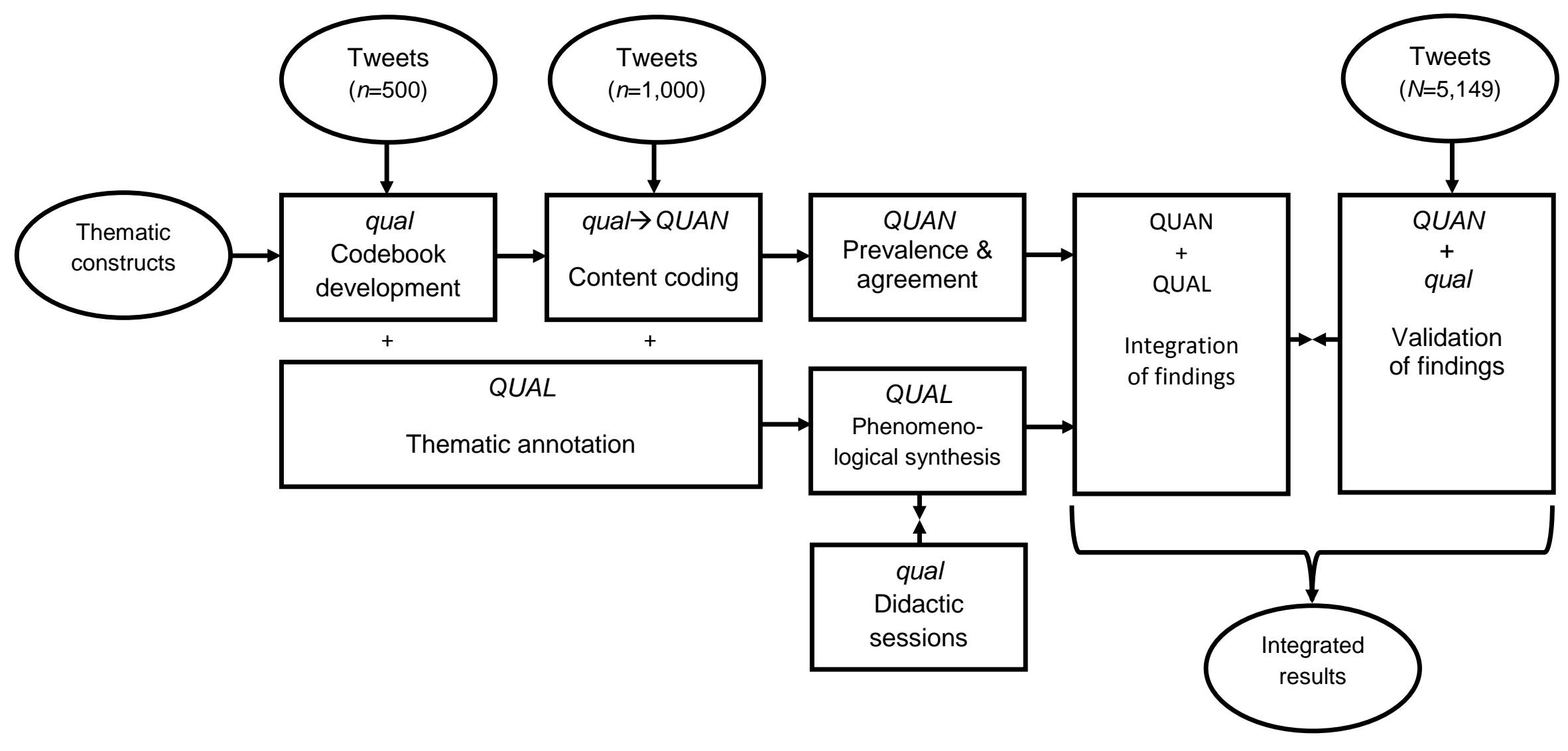

